\title{
Microscopic Engine Powered by Critical Demixing
}

\author{
Falko Schmidt, ${ }^{1,2,3}$ Alessandro Magazzù, ${ }^{1,2}$ Agnese Callegari, ${ }^{2}$ Luca Biancofiore, ${ }^{4}$ Frank Cichos, ${ }^{3}$ and Giovanni Volpe ${ }^{1,2, *}$ \\ ${ }^{1}$ Department of Physics, University of Gothenburg, 41296 Gothenburg, Sweden \\ ${ }^{2}$ Soft Matter Lab, Department of Physics and UNAM-National Nanotechnology Research Center, Bilkent University, \\ Ankara 06800, Turkey \\ ${ }^{3}$ Peter Debye Institute for Soft Matter Physics, Faculty of Physics and Earth Sciences, \\ Leipzig University, 04103 Leipzig, Germany \\ ${ }^{4}$ Department of Mechanical Engineering, Bilkent University, Ankara 06800, Turkey
}

(Received 31 July 2017; revised manuscript received 4 December 2017; published 9 February 2018)

\begin{abstract}
We experimentally demonstrate a microscopic engine powered by the local reversible demixing of a critical mixture. We show that, when an absorbing microsphere is optically trapped by a focused laser beam in a subcritical mixture, it is set into rotation around the optical axis of the beam because of the emergence of diffusiophoretic propulsion. This behavior can be controlled by adjusting the optical power, the temperature, and the criticality of the mixture.
\end{abstract}

DOI: 10.1103/PhysRevLett.120.068004

Introduction.-Engines are central to many natural and technological systems as devices capable of converting energy into mechanical work. During the last few decades, a lot of effort has gone into miniaturizing engines for applications in nanoscience and nanotechnology [1,2]. Differently from their macroscopic counterparts, microscopic engines are not completely deterministic, because they operate on energy scales where thermal fluctuations become relevant, and, therefore, need to be treated within stochastic thermodynamics [3,4].

Several approaches have been proposed to realize microscopic engines. Microrotators have been realized by transferring light's orbital and spin momentum to microparticles $[5,6]$ or by employing rotating magnetic fields [7-9]. Prototypes of microscopic heat engines have been realized exploiting the nucleation of vapor bubbles inside silicon microcavities, some with a working volume of only $0.6 \mathrm{~mm}^{3}$ [10-12]. More recently, optically trapped particles have been employed to reproduce microscopic versions of the Stirling and Carnot cycles, and to study their stochastic thermodynamic properties $[13,14]$. Also, a microscopic steam engine has been developed based on the periodic generation of cavitation bubbles by an optically trapped particle [15].

Here, we introduce a new mechanism to power a microscopic engine that relies on the local and reversible demixing of a critical mixture surrounding a microparticle. In particular, we show that an absorbing microsphere, suspended in a critical binary mixture and optically trapped, is able to perform rotational motion around the beam waist and to produce work thanks to the local demixing generated by the (slight) increase of the temperature of the solution when the particle approaches the focal point. The properties of this microscopic engine can be controlled by adjusting optical power, ambient temperature, and mixture criticality. Differently from the artificial microscopic engines mentioned above, the type of microscopic engine we propose here does not rely on the transfer of (angular) momentum from an external source (from circularly polarized light fields [5,6], high-order laser beams [16], or magnetic fields [8]) or on a flow of energy from a hot reservoir to a cold reservoir (microscopic Stirling [13], Carnot [14], and steam engines [15]). On the latter point, while in our realization of this type of engine a small temperature gradient is present to locally induce critical demixing, the presence of such temperature gradient is not generally required by the driving mechanism of this kind of engine, as it can be powered by other sources of critical demixing like $p \mathrm{H}$ or concentration gradients.

Results.-We consider an absorbing microsphere (silica with iron-oxide inclusions, $R=1.24 \pm 0.04 \mu \mathrm{m}$, Supplemental Material Sec. I and SEM image in Supplemental Fig. S1 [17]) optically trapped in a binary water-2,6-lutidine mixture at the critical lutidine mass fraction $c_{c}=0.286$ with a lower critical point at $T_{c} \approx$ $34^{\circ} \mathrm{C}$ [phase diagram in Fig. 1(a)] [18]. When $T<T_{c}$, the mixture is homogeneous and behaves like a normal fluid. As $T$ approaches $T_{c}$ [arrow in Fig. 1(a)], density fluctuations emerge. Finally, as $T$ exceeds $T_{c}$, the mixture demixes into water-rich and lutidine-rich phases.

The setup consists of an optical tweezers with a nearinfrared laser beam $(\lambda=976 \mathrm{~nm})$ built on a homemade inverted microscope; the sample-stage temperature $\left(T_{0}\right)$ is measured and stabilized with a feedback controller to $\pm 3 \mathrm{mK}$ (Supplemental Fig. S2 and Supplemental Sec. II [17]). The particle position is tracked by digital video microscopy at $296 \mathrm{fps}$. Rhodamine B, which is water soluble and fluoresces around the wavelength of $600 \mathrm{~nm}$, is added to the solution as an indicator of the phase separation 

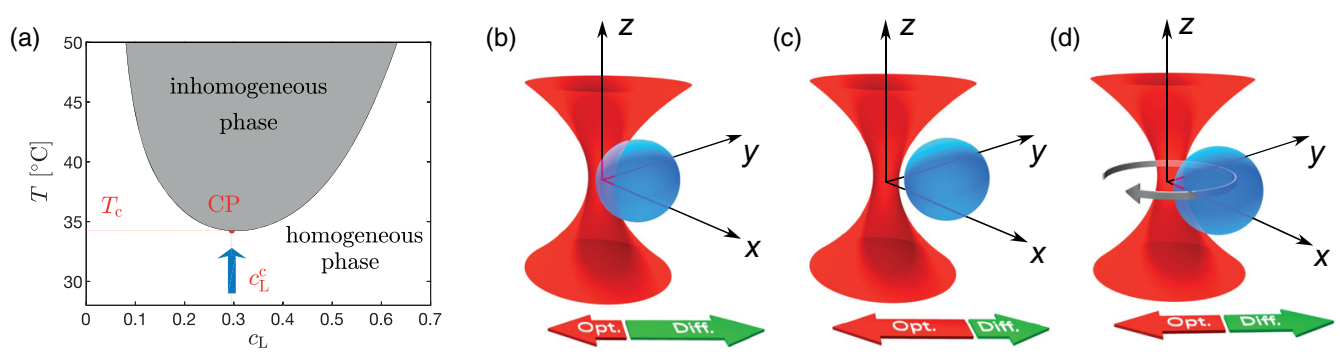

FIG. 1. Critical engine working principle. (a) Phase diagram of the water-2,6-lutidine mixture featuring a lower critical point $(C P)$ at the bottom of the coexistence line (black solid line). The system is prepared at the critical lutidine mass fraction $c_{c}=0.286$ and at a temperature $T_{0}$ significantly (some degrees) below the critical temperature $T_{c} \approx 34{ }^{\circ} \mathrm{C}$ (arrow). (b)-(d) An optically trapped microsphere experiences a harmonic restoring optical force that attracts it towards the center of the optical trap near the focal spot (red arrows). If the particle absorbs the illumination light, the temperature of the surrounding fluid increases generating an asymmetric temperature profile, which is hotter on the side of the particle closer to the focal spot. Because of the criticality of the mixture, the temperature gradient, in turn, generates a concentration gradient surrounding the particle and, eventually, a diffusiophoretic drift (green arrows). (b) When the particle is close to the focal spot, the diffusiophoretic drift dominates. (c) Instead, when the particle is far from the focal spot, the optical-force-induced drift dominates. (d) The equilibrium position along the radial direction lies where the optical and diffusiophoretic drifts balance each other and depends on the value of $T_{0}$ and on the light intensity; here the presence of small asymmetries in the temperature and demixing profile around the particle make the particle rotate around the optical axis (gray arrow).

of water and lutidine: the detected intensity increases with the local relative water content so that a higher intensity close to the particle's hydrophilic surface marks the region where the critical temperature $T_{c}$ has been exceeded.

The microsphere is attracted by optical forces (red arrows in Figs. 1(b)-1(d) and Supplemental Figs. S3(a)-S3(c) [17]) towards the optical trap center, near the focal spot. Because of the presence of iron-oxide inclusions, the microsphere partly absorbs the trapping light and converts it into heat, producing a local temperature increase (Supplemental Figs. S3(d)-S3(f) [17]). Since the particle side closer to the focal spot is hotter, an asymmetric temperature profile arises in the liquid surrounding the particle. Because of the mixture criticality, this temperature profile induces a concentration profile around the particle (Supplemental Figs. S3(g)-S3(i) [17]). Finally, the presence of this concentration profile generates a diffusiophoretic motion [19] of the particle away from the focal spot (green arrows in Figs. 1(b)-1(d) and Supplemental Figs. S3(a)-S3(c) [17]). Since the heating produced by the particle depends on the light intensity, it decreases as the particle moves radially away from the focal spot and, therefore, also the associated chemical gradient and diffusiophoretic motion decrease. Consequently, the particle settles off-axis, where the optical-force-induced drift is balanced by the diffusiophoretic drift, i.e., where the total radial force acting on the particle is zero (Fig. 1(d) and Supplemental Fig. S3(b) [17]). Importantly, there are small asymmetries in the composition of the particle (Supplemental Fig. S1 [17]) that induce asymmetries in the temperature and demixing profiles and, consequently, make the particle rotate around the optical axis (gray arrow in Fig. 1(d) and Supplemental Fig. S3(b) [17]). Several parameters play a role in the workings of this engine, in particular the trapping beam power $P$ and the ambient temperature $T_{0}$. While the critical engine presented here is powered by the localized critical demixing induced by a small temperature gradient, critical engines can be powered by other sources of critical demixing, e.g., localized $p \mathrm{H}$ or concentration gradients, that do not require temperature differences (Supplemental Fig. S5 [17]).

Laser power dependence.-We start by setting the ambient temperature to $T_{0}=26^{\circ} \mathrm{C}$. At low laser power ( $P=0.6 \mathrm{~mW}$ at the trap), the particle lingers around the optical trap center [solid line in Fig. 2(a)]. From the trajectory we calculate the velocity drift field, which indicates the direction and magnitude of the particle's velocity as a function of its position, and the differential cross-correlation function $\mathcal{D}_{x y}$, whose value indicates the magnitude of the rotational component of the force fields [20,21] (Supplemental Sec. VI [17]). The resulting drift field [white arrows in Fig. 2(b)] points towards the optical trap center and $\mathcal{D}_{x y}$ [Fig. 2(b)] is negligible, showing that there is no cross-correlation between the movement along the $x$ and $y$ directions, which means that nonconservative forces and therefore rotation are absent (i.e., the rotation rate $\Omega_{x y} \approx 0 \mathrm{rpm}$ ). All these results are consistent with the behavior of an optically trapped particle in a noncritical medium [22]; this is expected because at low power the heating is not enough to approach $T_{c}$ and, thus, to produce a demixing profile around the particle.

Even increasing the power up to $P=1.5 \mathrm{~mW}$, the particle is still constrained around the optical trap center [Fig. 2(d)], the drift field points towards the center of the trap [Fig. 2(e)], and nonconservative forces are negligible [Fig. 2(f)]. Also in this case, $\Omega_{x y} \approx 0 \mathrm{rpm}$. This is because the temperature increase due to light absorption is still insufficient to reach $T_{c}$.

Increasing the optical power to $P=2.1 \mathrm{~mW}$, the temperature of the solution surrounding the particle reaches $T_{c}$ leading to a local demixing [Fig. 2(g)]. 


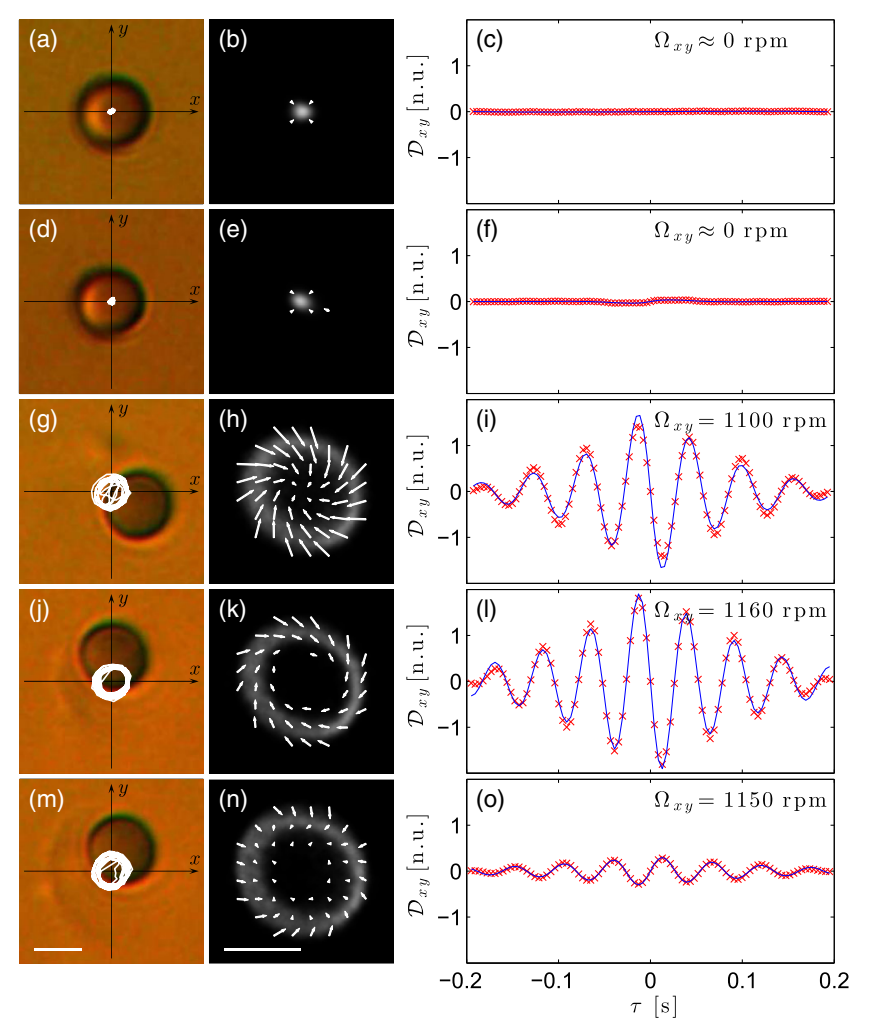

FIG. 2. Engine performance as a function of laser power. The ambient temperature of the sample is fixed at $T_{0}=26^{\circ} \mathrm{C}$, while the laser power at the optical trap is (a)-(c) $P=0.6$, (d)-(f) 1.5, (g)-(i) 2.1, (j)-(l) 2.7, and (m)-(o) $3.2 \mathrm{~mW}$. [(a),(d),(g),(j),(m)] Bright-field images of the particle with $0.6 \mathrm{~s}$ trajectories represented by white solid lines. [(b),(e),(h),(k),(n)] Velocity drift fields (white arrows) and particle position probability distributions (background color, brighter colors represent higher probability density). [(c),(f),(i),(l),(o)] Experimental (red symbols) and fitted (blue solid lines) differential cross-correlation functions $\mathcal{D}_{x y}$ in the $x y$ plane, from which the rotation rate of the particle $\Omega_{x y}$ can be obtained as fit parameter (reported in the right column). The white bars in (m) and (n) correspond to $1 \mu \mathrm{m}$. See also Supplemental Video 1 [17].

The brighter regions surrounding the particle in Fig. 2(g) correspond to water-rich regions, where the fluorescence of the Rhodamine $\mathrm{B}$ dye added to the solution is enhanced. The resulting concentration gradient induces a diffusiophoretic drift that pushes the particle radially outwards from the center, where it reaches a radial equilibrium position and starts rotating inside a toroidal region around the optical axis [Figs. 1(b)-1(d)]. This rotational motion can be seen from the particle trajectory [solid line in Fig. 2(g)] and, more quantitatively, from the drift field [white arrows in Fig. 2(h)], the particle probability distribution [background shading in Fig. 2(h)], and $\mathcal{D}_{x y}$ [Fig. 2(i)], which shows the sinusoidal behavior characteristic of rotational force fields. The resulting rotation of the particle is $\Omega_{x y}=1100 \mathrm{rpm}$. The rotational motion of the particle is due to structural asymmetries in the particle itself (e.g., in the distribution of the iron oxide and in its surface properties, Supplemental Fig. S1 [17]) and in the ensuing temperature gradient profile. Sometimes it can be observed that the rotation stops, the particle moves towards the center, and subsequently restarts rotating in the opposite direction; this behavior occurs whenever the Brownian fluctuations overcome the diffusiophoretic drift acting on the particles and orient the particle towards the optical trap center.

Increasing the power even further to $P=2.7 \mathrm{~mW}$, the particle performs constant revolutions around the optical trap center at $\Omega_{x y}=1160 \mathrm{rpm}$ without changing its direction during the measurement. Its radial equilibrium position reaches about $1 \mu \mathrm{m}$ [Fig. 2(j)]. The resulting drift field shows clearly a steady clockwise rotation [Fig. 2(k)] and $\mathcal{D}_{x y}$ increases its amplitude [Fig. 2(1)]. This rotation rate is comparable with those reached by micropumps and microrotors driven by optical vortices [23] while using only milliwatts (in contrast to several watts) of laser power.

As the power is further increased to $P=3.2 \mathrm{~mW}$, the particle not only rotates around but passes occasionally through the optical trap center and changes randomly its direction of rotation [Fig. 2(m)]. We determine $\Omega_{x y}=$ $1120 \mathrm{rpm}$. The higher laser power makes the temperature of the critical mixture surrounding the particle significantly exceed $T_{c}$, leading to a disruption of the balance between optical and diffusiophoretic drifts. Consequently, the particle is attracted towards the optical trap center before being pushed radially outwards by diffusiophoresis and returning to rotational motion. This leads to a partial disruption of the rotational component of the drift field [Fig. 2(n)] and to a decrease of $\mathcal{D}_{x y}$ [Fig. 2(o)].

When the power is further increased, the diffusiophoretic motion exceeds the optical trapping potential and therefore the particle escapes from the optical trap.

Ambient temperature dependence.-Fixing $P=2.7 \mathrm{~mW}$, the critical engine operation as a function of $T_{0}$ is optimal at $T_{0}=26^{\circ} \mathrm{C}$, where the particle steadily rotates around the optical trap center at $\Omega_{x y}=1160 \mathrm{rpm}$ (Supplemental Sec. IV and Supplemental Fig. S4 [17]).

Mixture criticality dependence.-To explore the dependence on the mixture criticality, we repeated the measurements at an off-critical lutidine mass fraction of 0.236 as a function of $P$ and $T_{0}$. We did not observe any rotational behavior even at high power $(P>6 \mathrm{~mW})$ and therefore concluded that the mixture criticality is an essential ingredient for the engine to work. Indeed, when the mixture is off-critical, water and lutidine undergo a more complex cooling and remixing process than when it is critical [24]; in particular, the presence of latent heat introduces a hysteresis cycle that slows down the remixing process when the temperature of the mixture drops below the binodal line (Supplemental Fig. S5(a) [17]). This prevents the engine from working because it is eventually obstructed by a bubble rich in one of the two phases created and stabilized around the particle so that the particle is 

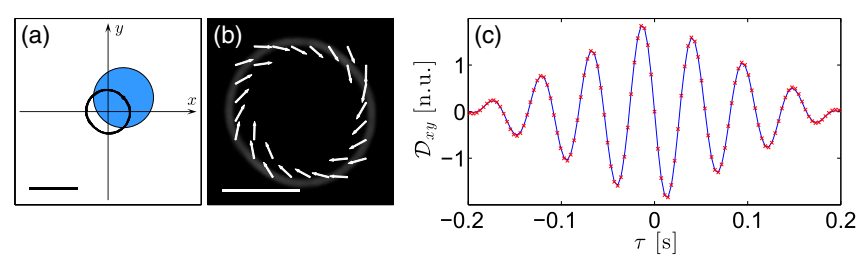

FIG. 3. Simulation of a critical engine. (a) Particle (blue circle) with corresponding $0.6 \mathrm{~s}$ trajectory (black line). The black bar corresponds to $1 \mu \mathrm{m}$. (b) Drift field (white arrows) and particle position distribution (background color, brighter colors represent higher probability density). The white bar corresponds to $1 \mu \mathrm{m}$. (c) Differential cross-correlation function $\mathcal{D}_{x y}$ in the $x y$ plane, from which the rotation rate is estimated to be $\Omega_{x y}=1200 \mathrm{rpm}$.

symmetrically surrounded by a single-phase liquid that inhibits diffusiophoretic propulsion so that the particle is optically trapped as usual in a homogenous fluid.

Particle size dependence. - The critical engine works also for smaller particles $(R=0.49 \pm 0.03 \mu \mathrm{m}$, Supplemental Figs. S6 and S7 [17]). The behavior is similar and the rotational rate higher $\left(\Omega_{x y}=4600 \mathrm{rpm}\right.$ at $P=1.5 \mathrm{~mW}$ and $T_{0}=23^{\circ} \mathrm{C}$, Supplemental Sec. V [17]). Furthermore, the critical engine working principle can be scaled down to the nanoscale as long as the slip velocity on the particle surface scales $\propto R^{-1}$; however, we expect the smooth rotational behavior to be disrupted by the increase of translational and rotational Brownian diffusion (Supplemental Secs. III and $\mathrm{V}[17])$.

Simulations.-To gain a deeper understanding of the mechanism responsible for the behavior observed experimentally, we also investigate the dynamics of the system numerically (Supplemental Sec. VII [17]). We consider an absorbing hydrophilic particle held by an optical trap. We assume that the particle is slightly asymmetric in its shape and material properties (nonuniform absorption and surface roughness). The optical forces are calculated using generalized Mie theory [22]. The temperature increase in the surroundings of the particle is calculated by determining the absorption of the laser light by the iron-oxide inclusions [25] and using the stationary heat equation to estimate the ensuing heat conduction [26]. This temperature increase causes a local demixing of the binary solution near the particle, resulting in an increase in the local concentration of water, because of the hydrophilicity of the particle's surface. The diffusiophoretic drift and torque acting on the particle are then calculated from the slip velocity field generated around the particle [19]; we remark that an alternative model that takes into account additionally advection has been proposed [27] and has been found to be in agreement with experiments in viscoelastic critical media [28]. Figure 3(a) shows a typical trajectory obtained from simulations, which features the rotational motion at a rotational rate $\Omega_{x y}=1200 \mathrm{rpm}$, in quantitative agreement with the experiments [compare with Figs. 2(j) and S4(g)]; the corresponding drift field [Fig. 3(b)] and $\mathcal{D}_{x y}$ [Fig. 3(c)]
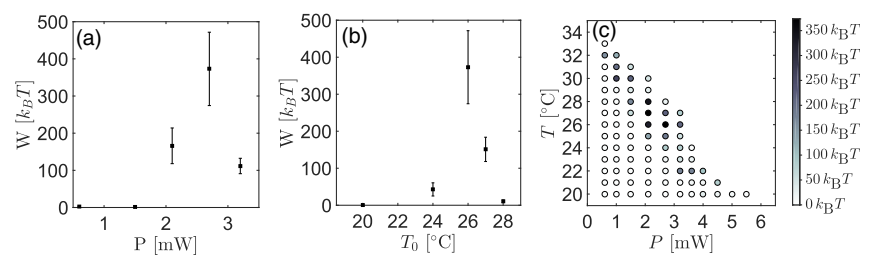

FIG. 4. Work dependence on laser power and ambient temperature. Work $W$ as a function of (a) laser power $P\left(T_{0}=26^{\circ} \mathrm{C}\right)$ and (b) ambient temperature $T_{0}(P=2.7 \mathrm{~mW})$. (c) Work as a function of $P$ and $T_{0}$. The circles represent the performed measurements. $W$ features a maximum for $P=2.7 \mathrm{~mW}$ and $T_{0}=26^{\circ} \mathrm{C}$.

are also in quantitative agreement with the experiments [compare with Figs. 2(k) and S4(h), and with Figs. 2(l) and S4(i), respectively].

Performance.-The rotational motion of the particle around the beam waist is intrinsically nonconservative and corresponds to a rotational force field. Through the analysis of $\mathcal{D}_{x y}$ (Supplemental Sec. VI [17]), we evaluate the work performed by the particle during a single rotation about the $z$ axis as [29]

$$
W=2 \pi \frac{\Omega_{x y}}{\omega_{\rho}} k_{B} T,
$$

where $W$ is directly proportional to the rotation rate $\Omega_{x y}$ associated with the nonconservative force field and inversely proportional to the transverse relaxation frequency $\omega_{\rho}$ of the optical trap. The efficiency of the engine is given by $\eta_{\text {eff }}=\left[\left(\Omega_{x y} W\right) / P\right]$. The value of $W$ is plotted as a function of $P$ and $T_{0}$ in Fig. 4. The maximum of $W=373 k_{B} T$ and $\eta_{\text {eff }}=1.16 \times 10^{-14}$ is reached for $P=2.7 \mathrm{~mW}$ and $T_{0}=26^{\circ} \mathrm{C}$ at $\Omega_{x y}=1160 \mathrm{rpm}$. The amount of work performed by this critical engine exceeds that of colloidal heat engines such as the Brownian Carnot engine $\left(W_{\max }=5 k_{B} T\right)$ [14] and the micrometer-sized heat engine $\left(W_{\max }=0.3 k_{B} T\right)$ [13]. Its efficiency is comparable to the efficiency of a rotating object driven by the transfer of the angular momentum from a circularly polarized beam $\left(\eta_{\text {eff }} \sim 10^{-14}\right)$ [6]. Even though in our case the work done by the particle is immediately dissipated as heat into the fluid, it remains in principle accessible, e.g., by attaching a load to the particle.

Discussion.-We have realized a micron-sized critical engine that can extract work from the criticality of a system. In our realization, the efficiency of the critical engine can be tuned by adjusting the laser power, the ambient temperature, or the mixture criticality. Compared to other micronsized engines developed in the last years [13-15], this critical engine has the advantages of not relying on the transfer of external angular momentum and of working at temperatures close to room temperature. It is in principle possible to control the sense of rotation of the critical engine by designing chiral particles with a controllable absorption profile. The work performed per cycle exceeds 
that of other microscopic heat engines by orders of magnitude [13,14]; its efficiency is comparable to micron-sized engines driven by transfer of optical (angular) momentum [16], while much higher efficiencies can be achieved with alternative mechanisms such as the thermocapillary effect [30]. Moreover, with only a few milliwatts of laser power the engine reaches rotation rates of $1200 \mathrm{rpm}$ for $1.24 \mu \mathrm{m}$-radius particles and of $4600 \mathrm{rpm}$ for $0.49 \mu \mathrm{m}$ radius particles; these values are much larger than those of micron-sized heat engines, which reach rates of about $10 \mathrm{rpm}$ [13]. Furthermore, in experiments with optical vortices similar rotation rates can only be achieved at powers on the order of watts $[23,31,32]$.

Importantly, since many natural and artificial systems are tuned near criticality, the critical engine working principle can be exploited in diverse applications and used to explain how natural phenomena work (e.g., molecular motors acting within a cellular membrane). In fact, it can exploit any order parameter tuned near criticality in a given system, e.g., $p \mathrm{H}$-value or particle concentration [33]. This also means that the critical engine operation can arise also in the absence of temperature gradients, as long as other gradients capable of tuning the system criticality are present. Since phase separations have been already widely found inside the human body $[34,35]$ and some of them are known to be sources of diseases such as protein condensation [36], new biocompatible engines can be designed based on our critical engine that could be able to perform medical surgeries noninvasively such as the treatment of arteriosclerosis.

We thank Andrea Gambassi and Klaus Kroy for fruitful discussions about the theory, and Christian Schmidt from microParticles $\mathrm{GmbH}$ for insightful discussions about the particles. This work was partially supported by the ERC Starting Grant ComplexSwimmers (Grant No. 677511) and by Vetenskapsrådet (Grant No. 2016-03523). A. C. acknowledges partial support from Tübitak (Grant No. 115F401).

*giovanni.volpe@physics.gu.se

[1] W. R. Browne and B. L. Feringa, Nat. Nanotechnol. 1, 25 (2006).

[2] L. K. E. A. Abdelmohsen, F. Peng, Y. Tu, and D. A. Wilson, J. Mater. Chem. B 2, 2395 (2014).

[3] U. Seifert, Rep. Prog. Phys. 75, 126001 (2012).

[4] I. A. Martínez, É. Roldán, L. Dinis, and R. A. Rica, Soft Matter 13, 22 (2017).

[5] N. B. Simpson, K. Dholakia, L. Allen, and M. J. Padgett, Opt. Lett. 22, 52 (1997).

[6] M. E. J. Friese, T. A. Nieminen, N. R. Heckenberg, and H. Rubinsztein-Dunlop, Nature (London) 394, 348 (1998).

[7] S. L. Biswal and A. P. Gast, Anal. Chem. 76, 6448 (2004).

[8] A. Ghosh and P. Fischer, Nano Lett. 9, 2243 (2009).
[9] B. Eickenberg, F. Wittbracht, P. Stohmann, J.-R. Schubert, C. Brill, A. Weddemann, and A. Hutten, Lab Chip 13, 920 (2013).

[10] J. Kao, X. Wang, J. Warren, J. Xu, and D. Attinger, J. Micromech. Microeng. 17, 2454 (2007).

[11] C. Lee, M. Liamini, and L. G. Frechette, J. Microelectromech. Syst. 20, 326 (2011).

[12] S. Percy, C. Knight, S. McGarry, A. Post, T. Moore, and K. Cavanagh, Thermal Energy Harvesting for Application at MEMS Scale, Springer Briefs in Electrical and Computer Engineering, 1st ed. (Springer-Verlag New York, 2014).

[13] V. Blickle and C. Bechinger, Nat. Phys. 8, 143 (2012).

[14] I. A. Martinez, E. Roldan, L. Dinis, D. Petrov, J. M. R. Parrondo, and R. A. Rica, Nat. Phys. 12, 67 (2016).

[15] P. A. Quinto-Su, Nat. Commun. 5, 5889 (2014).

[16] H. He, N. R. Heckenberg, and H. Rubinsztein-Dunlop, J. Mod. Opt. 42, 217 (1995).

[17] See Supplemental Material at http://link.aps.org/ supplemental/10.1103/PhysRevLett.120.068004 for recorded brightfield images of the engine as a function of laser power.

[18] C. A. Grattoni, R. A. Dawe, C. Y. Seah, and J. D. Gray, J. Chem. Eng. Data 38, 516 (1993).

[19] A. Würger, Phys. Rev. Lett. 115, 188304 (2015).

[20] G. Volpe and D. Petrov, Phys. Rev. Lett. 97, 210603 (2006).

[21] G. Volpe, G. Volpe, and D. Petrov, Phys. Rev. E 76, 061118 (2007).

[22] P. H. Jones, O. M. Maragò, and G. Volpe, Optical Tweezers: Principles and Applications (Cambridge University Press, Cambridge, England, 2015).

[23] K. Ladavac and D. G. Grier, Opt. Express 12, 1144 (2004).

[24] E. D. Siggia, Phys. Rev. A 20, 595 (1979).

[25] E. J. G. Peterman, F. Gittes, and C. F. Schmidt, Biophys. J. 84, 1308 (2003).

[26] D. Rings, R. Schachoff, M. Selmke, F. Cichos, and K. Kroy, Phys. Rev. Lett. 105, 090604 (2010).

[27] S. Samin and R. van Roij, Phys. Rev. Lett. 115, 188305 (2015).

[28] J. R. Gomez-Solano, A. Blokhuis, and C. Bechinger, Phys. Rev. Lett. 116, 138301 (2016).

[29] G. Pesce, G. Volpe, A. Imparato, G. Rusciano, and A. Sasso, J. Opt. 13, 044006 (2011).

[30] C. Maggi, F. Saglimbeni, M. Dipalo, F. De Angelis, and F. Di Leonardo, Nat. Commun. 6, 7855 (2015).

[31] S. H. Tao, X. C. Yuan, J. Lin, X. Peng, and H. B. Niu, Opt. Express 13, 7726 (2005).

[32] D. G. Grier, Nature (London) 424, 810 (2003).

[33] E. Tagliazucchi, D. R. Chialvo, M. Siniatchkin, E. Amico, J.-F. Brichant, V. Bonhomme, Q. Noirhomme, H. Laufs, and S. Laureys, J. R. Soc. Interface 13, 20151027 (2016).

[34] I. Digel, C. Maggakis-Kelemen, K. F. Zerlin, P. Linder, N. Kasischke, P. Kayser, and G. M. Artmann, Biophys. J. 91, 3014 (2006).

[35] X. M. Liu, L. S. Wang, L. Wang, J. Huang, and C. He, Biomaterials 25, 5659 (2004).

[36] W. M. Jacobs and D. Frenkel, Biophys. J. 112, 683 (2017). 